\title{
Blood markers in remote ischaemic conditioning for acute ischaemic stroke: data from the REmote ischaemic Conditioning After Stroke Trial
}

\author{
Jason P. Appleton ${ }^{1,2}$ (6) | Saoirse E. O'Sullivan ${ }^{3}$ @ | Amanda Hedstrom ${ }^{3}$ | Jane A. May ${ }^{1}$ |

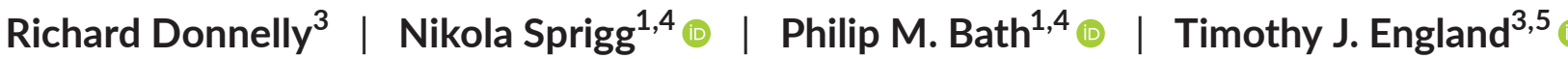

${ }^{1}$ Stroke Trials Unit, Division of Clinical Neuroscience, University of Nottingham, Nottingham, UK

${ }^{2}$ Stroke, Queen Elizabeth Hospital Birmingham, University Hospitals Birmingham NHS Foundation Trust, Birmingham, UK

${ }^{3}$ Division of Medical Sciences and GEM, School of Medicine, Vascular Medicine, University of Nottingham, Derby, UK

${ }^{4}$ Stroke, City Hospital, Nottingham University Hospitals NHS Trust, Nottingham, UK

${ }^{5}$ University Hospitals of Derby and Burton NHS Foundation Trust, Royal Derby Hospital, Derby, UK

Correspondence

Timothy J England, Division of Medical Sciences and GEM, Vascular Medicine,

School of Medicine, University of

Nottingham, Derby, UK.

Email: timothy.england@nottingham.ac.uk

Funding information

Health Technology Assessment

Programme, Grant/Award Number: 10/104/24; British Medical Association, Grant/Award Number: Vera Down Grant; British Heart Foundation, Grant/Award Number: CS/14/4/ 30972

\begin{abstract}
Background and purpose: Remote ischaemic per-conditioning (RIC) is neuroprotective in experimental ischaemic stroke. Several neurohumoral, vascular and inflammatory mediators are implicated. The effect of RIC on plasma biomarkers was assessed using clinical data from the REmote ischaemic Conditioning After Stroke Trial (RECAST-1).

Methods: RECAST-1 was a pilot sham-controlled blinded trial in 26 patients with ischaemic stroke, randomized to receive four 5-min cycles of RIC within $24 \mathrm{~h}$ of ictus. Plasma taken pre-intervention, immediately post-intervention and on day 4 was analysed for nitric oxide (nitrate/nitrite) using chemiluminescence and all other biomarkers by multiplex analysis. Biomarkers were correlated with clinical outcome (day 90 National Institutes of Health Stroke Scale, modified Rankin Scale, Barthel index).

Results: Remote ischaemic per-conditioning reduced serum amyloid protein (SAP) and tissue necrosis factor $\alpha$ (TNF- $\alpha$ ) levels from pre- to post-intervention ( $n=13$, two-way ANOVA, $p<0.05)$. Overall $(n=26)$, increases in SAP pre- to post-intervention and pre-intervention to day 4 were moderately correlated with worse day 90 clinical outcomes. No consistent significant changes over time, or by treatment, or correlations with outcome were seen for other biomarkers.

Conclusions: Remote ischaemic per-conditioning reduced SAP and TNF- $\alpha$ levels from pre- to post-intervention. Increases in plasma levels of SAP were associated with worse clinical outcomes after ischaemic stroke. Larger studies assessing biomarkers and the safety and efficacy of RIC in acute ischaemic stroke are warranted to further understand these relationships.
\end{abstract}

KEYWORDS

biomarkers, ischaemic stroke, nitric oxide, remote ischaemic conditioning, serum amyloid protein

Abbreviations: A2 M, alpha-2 macroglobulin; BI, Barthel index; BP, blood pressure; CI, confidence interval; GCSF, granulocyte colony-stimulating factor; IL, interleukin; mRS, modified Rankin Scale; NIHSS, National Institutes of Health Stroke Scale; NO, nitric oxide; RECAST, REmote ischaemic Conditioning After Stroke Trial; RIC, remote ischaemic conditioning; SAP, serum amyloid protein; TNF- $\alpha$, tissue necrosis factor $\alpha$.

This is an open access article under the terms of the Creative Commons Attribution License, which permits use, distribution and reproduction in any medium, provided the original work is properly cited.

(c) 2020 The Authors. European Journal of Neurology published by John Wiley \& Sons Ltd on behalf of European Academy of Neurology 


\section{INTRODUCTION}

Remote ischaemic per-conditioning (RIC)-inducing ischaemia/ reperfusion distant to the brain-in experimental ischaemic stroke is neuroprotective [1-3]. Several neurohumoral, vascular and inflammatory mediators are implicated, but the underlying mechanisms have not been elucidated. Animal models have demonstrated several inflammatory, neuroprotective and vascular biomarkers that may be influenced by RIC, primarily in the setting of myocardial ischaemia and infarction [1].

There is a paucity of data on the effects of RIC on potential mechanistic pathways in human patients with acute ischaemic stroke. Plasma heat shock proteins (HSP) 27, 60 and 70, C-reactive protein, S100- $\beta$, matrix metalloproteinase-9 (MMP-9), troponin T and endocannabinoid data have been reported in acute ischaemic stroke patients undergoing RIC in the REmote ischaemic Conditioning After Stroke Trial (RECAST-1) [4]. There were significant increases in total and phosphorylated HSP 27 in the RIC group, whilst the other biomarker levels did not change. Whether other inflammatory, neuroprotective or vascular markers are influenced by RIC in acute ischaemic stroke is unknown.

Several inflammation-related biomarkers including adipsin [5] $\alpha 2$-macroglobulin (A2 M), serum amyloid protein (SAP) [6] and E-selectin [7] have been associated with clinical outcome in stroke patients. Whether these or other elements of inflammatory pathways are influenced by RIC is unclear. Similarly, biomarkers contributing to vascular or neuroprotective pathways-vascular endothelial growth factor (VEGF) [8] von Willebrand factor (vWF) [9] or nitric oxide (NO) - may have roles in RIC $[2,10]$ but have not been assessed in humans with acute ischaemic stroke.

Given the limited data regarding RIC and biomarkers in ischaemic stroke patients, in exploratory and hypothesis-generating analyses the effect of RIC on plasma biomarkers and the correlation of these biomarkers with clinical outcomes in acute ischaemic stroke patients in RECAST-1 were assessed.

\section{METHODS}

\section{Population}

RECAST-1 was a pilot, single-centre, randomized, blinded, sham-controlled trial in 26 patients with ischaemic stroke, who were randomized to receive four 5-min cycles of RIC or sham within $24 \mathrm{~h}$ of onset. The main results of the trial have been published: RIC was well tolerated, appeared safe and feasible in ischaemic stroke patients, and increased plasma HSP 27 [4]. In brief RECAST-1 recruited 26 adult patients from Derby Teaching Hospitals NHS Foundation Trust, UK, with ischaemic stroke within $24 \mathrm{~h}$ of ictus with arm and/or leg weakness. The intervention comprised four cycles of intermittent upper limb ischaemia with $5 \mathrm{~min}$ inflation $20 \mathrm{mmHg}$ above the systolic blood pressure and $5 \mathrm{~min}$ deflation using a manual blood pressure cuff on the non-paretic arm. The sham group received cuff inflation to $30 \mathrm{mmHg}$ only. The trial was registered (ISRCTN 86672015).

\section{Blood samples}

Venous blood samples were taken pre-intervention, immediately post-intervention and on day 4 . These samples were centrifuged and the supernatant was stored at $-80^{\circ} \mathrm{C}$ and later thawed for batch analysis. All biomarkers, except for NO, were analysed by enzymelinked immunosorbent assay.

\section{Inflammation-related blood biomarkers}

The MILLIPLEX panel (HCYTOMAG-60 K-08) was performed per the manufacturer's instructions to detect changes in granulocyte colonystimulating factor (GCSF), interleukin-1 $\alpha$ (IL-1 $\alpha$ ), IL-1 $\beta$, IL-1 receptor antagonist (RA), IL-6, IL-10 and tissue necrosis factor $\alpha$ (TNF- $\alpha$ ) in plasma using the Luminex ${ }^{\circledR} \times \mathrm{XMAP}{ }^{\circledR}$ technology. HCVD3MAG-67 K-05 was used to detect adipsin, A2 M and SAP. E-selectin was measured using HCVD4MAG-67 K-02.

\section{Neuroprotective and vascular blood biomarkers}

Nitric oxide $\left(\mathrm{NO}_{x}\right)$ in the form of nitrate $\left(\mathrm{NO}_{3}\right)$ and nitrite $\left(\mathrm{NO}_{2-}\right)$ was measured in plasma samples using chemiluminescence (Sievers 280 nitric oxide analyser, Analytix Ltd). Nitrate and nitrite are reduced to NO, which reacts with ozone to produce light detected by a sensitive photomultiplier tube. NO analyser calibration was performed by injecting sodium nitrate standards ranging from $100 \mathrm{nM}$ to $100 \mu \mathrm{M}$ in concentration. Plasma samples, deproteinized with ethanol, were injected in duplicate and the concentrations of plasma $\mathrm{NO}_{x}$ were calculated from the calibration curve using the liquid programme installed on a dedicated laptop.

The MILLIPLEX panel (HCYTOMAG-60 K-08) also included measuring VEGF-A in plasma using the Luminex ${ }^{\circledR} \times \mathrm{xMAP} \otimes$ technology as per the manufacturer's instructions. vWF was measured using HCVD3MAG-67 K-05.

\section{Clinical outcomes}

Clinical outcome measures were assessed at day 90: National Institutes of Health Stroke Scale (NIHSS), a measure of stroke severity; modified Rankin Scale (mRS), functional outcome; Barthel index (BI), a measure of disability; and the Mini-Mental State Examination (MMSE), a measure of cognition.

\section{Statistics}

The sample size for RECAST-1 was calculated for treatment tolerability: 26 participants were studied assuming a meaningful delivery of three of the four 5-min cycles of RIC with $90 \%$ power to reject the null hypothesis that intervention and sham were equally 
tolerated. The present analyses are exploratory and hypothesisgenerating. Data are number (\%), mean (standard deviation) and median (interquartile range). Baseline characteristics were compared by chi-squared test, Kruskal-Wallis test or one-way ANOVA as appropriate. Changes in biomarkers over time were assessed using two-way ANOVA with resultant mean difference (MD) and 95\% confidence interval (CI). Biomarkers were correlated with clinical outcomes at day 90 using Pearson's correlation coefficient. No adjustment was made for multiple testing. Significance was set at $p<0.05$. Analyses were performed using SPSS version 24 and Prism version 7.

\section{RESULTS}

In total, 26 participants were recruited with a mean age of 76 (10.5) years, 35\% female, with moderate severity ischaemic strokes (NIHSS 6) and were randomized at $15.8 \mathrm{~h}$ after stroke onset. Bloods were taken before the intervention, immediately after and on day 4. Baseline characteristics were well balanced between treatment groups, although the sham group had more diabetes mellitus (Table 1).

\section{Inflammation-related blood biomarkers}

Serum amyloid protein levels reduced from pre- to post-intervention in those randomized to RIC ( $n=13$, two-way ANOVA, MD 10.47, 95\% $\mathrm{Cl} 0.30-20.64, p<0.05$ ) but did not in those randomized to sham (Figure 1). Levels of TNF- $\alpha$ also fell from pre- to post-intervention in those randomized to RIC ( $n=13$, two-way ANOVA, MD 3.58, 95\% $\mathrm{Cl} 0.40-6.77, p<0.05)$. No significant changes over time, or by treatment, were seen for adipsin, A2 M, GCSF-A or E-selectin (Figure 1).

IL-1RA, an immunomodulatory cytokine that inhibits the actions of IL- $1 \alpha$ and IL- $1 \beta$, fell from pre- to post-intervention in the sham group ( $n=13$, two-way ANOVA, MD 17.11, 95\% Cl 2.46-31.77, $p<0.05$ ) but did not change in those randomized to RIC (Figure 2). No significant changes pre- to post-intervention or to day 4 , or by treatment allocation, were seen for the other interleukins measured (Figure 2).

\section{Neuroprotective and vascular blood biomarkers}

Plasma NO, vWF or VEGF levels did not change over time or differ between treatment groups (Figure 3).
TABLE 1 Baseline characteristics of RECAST-1 participants

\begin{tabular}{|c|c|c|c|c|}
\hline & All & RIC & Sham & $\begin{array}{l}p \text { value } \\
\text { (RIC vs. } \\
\text { sham) }\end{array}$ \\
\hline Patients & 26 & 13 & 13 & \\
\hline Age (years) & $76.2(10.5)$ & $74.7(10.8)$ & $77.7(10.4)$ & 0.48 \\
\hline Sex, female & 9 (34.6\%) & $5(38.5 \%)$ & $4(30.8 \%)$ & 0.68 \\
\hline $\begin{array}{l}\text { Onset to randomization } \\
\text { (h) }\end{array}$ & $15.8(6.2)$ & $16.3(5.9)$ & $15.3(6.6)$ & 0.67 \\
\hline Systolic BP (mmHg) & $159.2(25.0)$ & $153.7(18.9)$ & $164.7(29.6)$ & 0.27 \\
\hline Diastolic BP (mmHg) & $82.9(10.6)$ & $81.5(7.5)$ & $84.3(13.7)$ & 0.53 \\
\hline Heart rate (bpm) & $76.3(15.2)$ & $78.5(13.1)$ & $74.1(17.3)$ & 0.47 \\
\hline Premorbid mRS [/6] & $0.0[0.0,1.0]$ & $0.0[0.0,0.5]$ & $0.0[0.0,2.0]$ & 0.45 \\
\hline NIHSS [/42] & $6[3.7,11]$ & $6[3.5,12]$ & $5[3.9,9.5]$ & 0.72 \\
\hline TACS & $8(30.8 \%)$ & $4(30.8 \%)$ & $4(30.8 \%)$ & 1.0 \\
\hline \multicolumn{5}{|l|}{ Past medical history } \\
\hline Hypertension & $14(53.8 \%)$ & $5(38.5 \%)$ & $9(69.2 \%)$ & 0.12 \\
\hline Hyperlipidaemia & $12(46.2 \%)$ & $5(38.5 \%)$ & 7 (53.8\%) & 0.43 \\
\hline Diabetes & $5(19.2 \%)$ & $0(0 \%)$ & $5(38.5 \%)$ & 0.01 \\
\hline Atrial fibrillation & $8(30.8 \%)$ & $3(23.1 \%)$ & 5 (38.5\%) & 0.40 \\
\hline Previous stroke & $5(19.2 \%)$ & $1(7.7 \%)$ & $4(30.8 \%)$ & 0.14 \\
\hline Previous TIA & $3(11.5 \%)$ & 2 (15.4\%) & $1(7.7 \%)$ & 0.54 \\
\hline $\begin{array}{l}\text { Ischaemic heart } \\
\text { disease }\end{array}$ & $7(26.9 \%)$ & 2 (15.4\%) & $5(38.5 \%)$ & 0.19 \\
\hline
\end{tabular}

Notes:: Comparison by chi-squared test, Kruskal-Wallis test or one-way ANOVA. Data are $n$ (\%), mean (standard deviation) or median [interquartile range].

Abbreviations: BP, blood pressure; bpm: beats per minute; mRS, modified Rankin Scale; NIHSS, National Institutes of Health Stroke Scale; RIC, remote ischaemic conditioning; TACS, total anterior circulation syndrome; TIA, transient ischaemic attack. 


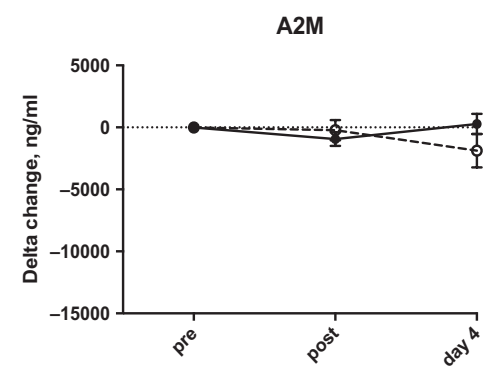

GCSF

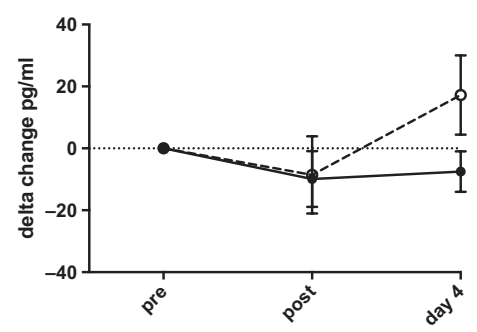

Adipsin

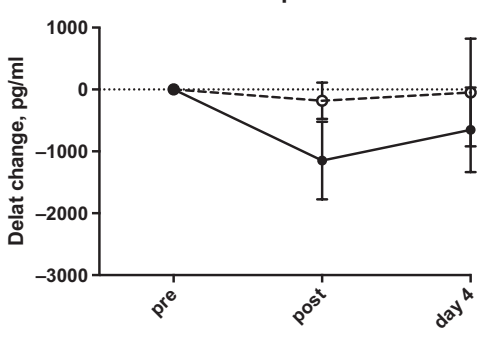

E-selectin

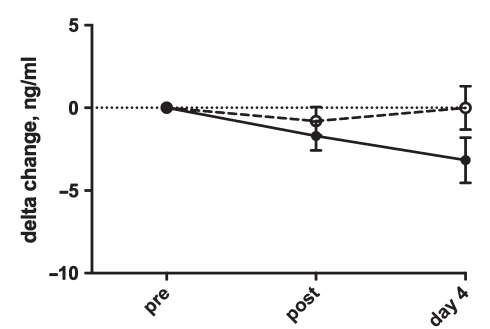

SAP

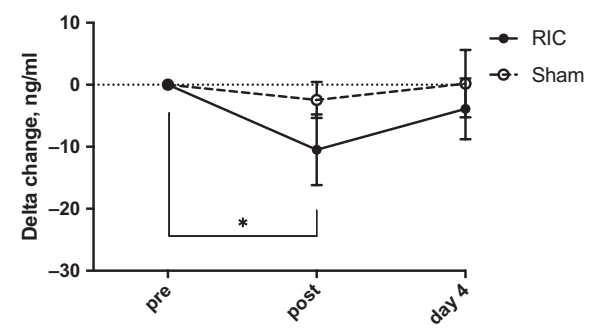

TNF alpha

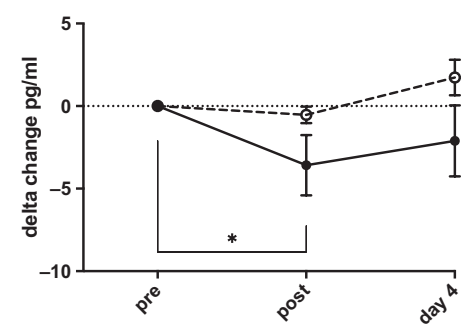

FIGURE 1 Effect of RIC on inflammation-related blood biomarkers. A2 M, alpha-2 macroglobulin; GCSF, granulocyte colony-stimulating factor; SAP, serum amyloid protein; TNF, tissue necrosis factor. ${ }^{*} p<0.05$ in RIC group over time, two-way ANOVA.
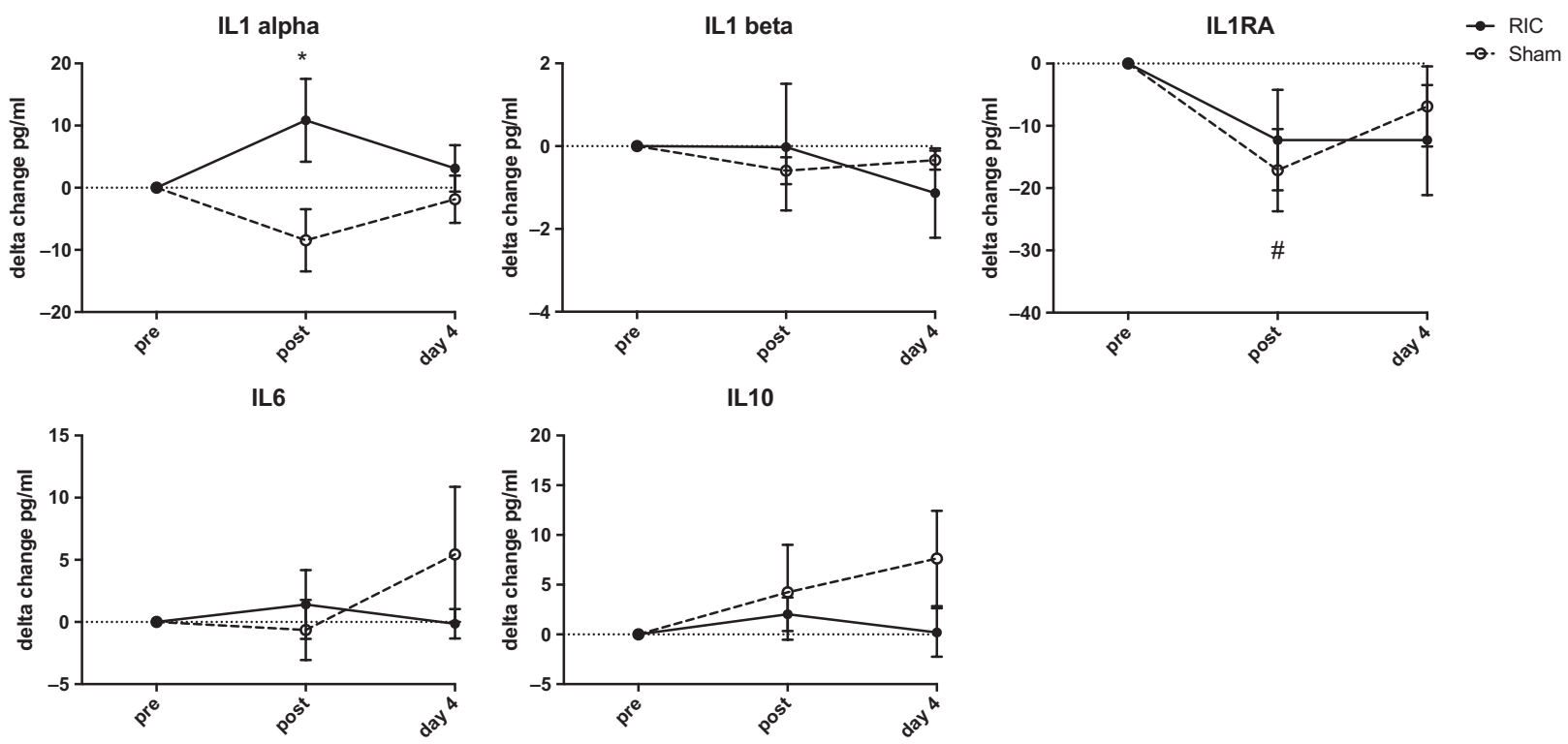

FIGURE 2 Effect of RIC on interleukins. IL, interleukin; IL-1RA, IL-1 receptor antagonist. * $p<0.05$ between groups; \#p < 0.05 in sham group over time, two-way ANOVA.

\section{Association between absolute biomarker levels and clinical outcomes}

Overall, higher S100- $\beta$ levels at pre-intervention, post-intervention and day 4 were significantly moderately correlated with worse NIHSS scores at day 90, but not mRS or BI (Table 2, Figure S1). Higher IL-1RA levels at day 4 were significantly moderately correlated with worse day 90 clinical outcomes (NIHSS, $r=0.410$, $p=0.038 ; \mathrm{mRS}, r=0.455, p=0.019 ; \mathrm{BI}, r=-0.387, p=0.051$; Figure S2). Higher IL-6 levels post-intervention were significantly strongly correlated with improved day 90 clinical outcomes (mRS, $r=-0.640, p=0.025 ; \mathrm{BI}, r=0.651, p=0.022$ ); this correlation was mainly seen in those randomized to sham, not RIC (Tables S1 and S2). Inconsistently, higher IL-6 levels at day 4 were significantly strongly correlated with worse NIHSS scores overall. Conversely, higher IL-10 levels at day 4 were significantly moderately and strongly correlated with worse day 90 clinical outcomes (NIHSS, $r=0.702, p<0.001 ; \mathrm{mRS}, r=0.493, p=0.023$; BI, -0.444 , $p=0.044$; Figure S2). No other consistently significant correlations were noted for other biomarkers (Tables 2, S1 and S2). 
NOx

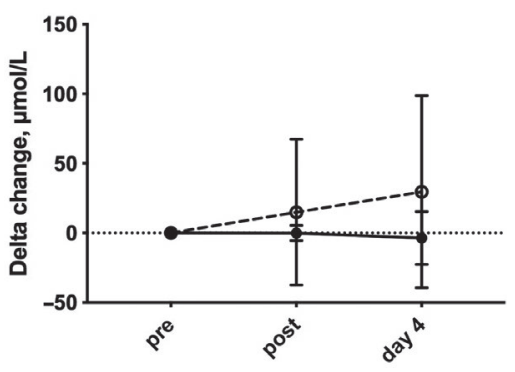

vWF

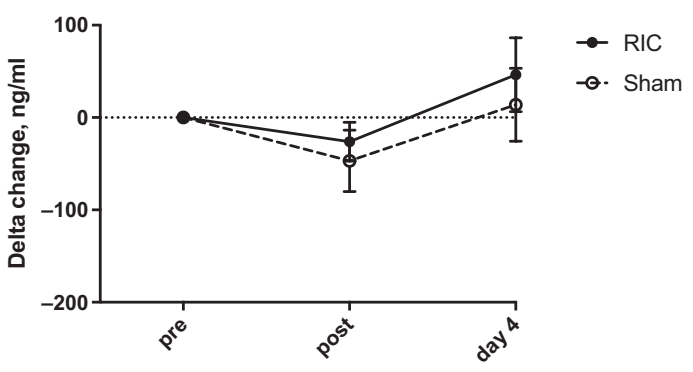

FIGURE 3 Effect of RIC on neuroprotective and vascular blood biomarkers. NOx, nitrate/nitrite; vWF, von Willebrand factor; VEGF, vascular endothelial growth factor.

\section{Association between change in biomarker levels and clinical outcomes}

Overall, increases in SAP pre- to post-intervention and pre-intervention to day 4 were significantly moderately correlated with worse day 90 clinical outcomes (NIHSS, $r=0.400, p=0.043 ; \mathrm{mRS}, r=0.505$, $p=0.008 ; \mathrm{BI}, r=-0.439, p=0.025 ; \mathrm{MMSE}, r=-0.395, p=0.051$ Table 3, Figure S3a,b). These significant correlations were seen in those randomized to sham but not in those randomized to RIC (Tables S3 and S4). Overall, an increase in A2 M pre-intervention to day 4 was weakly and moderately correlated with improved day $90 \mathrm{mRS}(r=-0.359$, $p=0.07)$ and MMSE $(r=0.465, p=0.019)$ respectively. An increase in HSP 60 pre-intervention to day 4 was moderately and strongly correlated with worse clinical outcomes at day 90 (NIHSS, $r=0.641$, $p=0.007 ; \mathrm{mRS}, r=0.500, p=0.049 ; \mathrm{BI}, r=-0.523, p=0.038 ;$ Figure S4). Overall, an increase in IL-6 pre- to post-intervention was strongly correlated with improved clinical outcomes at day 90 (NIHSS, $r=-0.584$, $p=0.059 ; \mathrm{mRS}, r=-0.677, p=0.022 ; \mathrm{BI}, r=0.690, p=0.019$ ), which was mainly seen in those randomized to sham, not RIC (Tables S3 and S4). In contrast, an increase in IL-6 over 4 days was strongly correlated with worse NIHSS and non-significantly weakly correlated with worse $\mathrm{mRS}$ and $\mathrm{BI}$. A similar picture was seen for IL-10 with increasing levels over 4 days being associated with a worse NIHSS ( $r=0.592, p=0.026)$. An increase in MMP-9 pre- to post-intervention was significantly moderately correlated with worse clinical outcomes at day 90 (Figure S5); no such association was seen pre-intervention to day 4. An increase in S100- $\beta$ pre-intervention to day 4 was moderately correlated with higher NIHSS scores at day 90. No significant correlations with outcome were seen for adipsin, GCSF-A, E-selectin, TNF- $\alpha$ or other HSPs (Table 3).
An increase in NO levels pre-intervention to day 4 had a tendency towards being weakly correlated with worse clinical outcomes at day 90 (NIHSS, $r=0.372, p=0.062$; mRS, $r=0.334, p=0.10$; $\mathrm{BI}, r=-0.355, p=0.08$, Table 3, Figure S6). Increases in VEGF levels pre- to post-intervention and pre-intervention to day 4 were non-significantly weakly correlated with better clinical outcomes. No significant correlations were noted for vWF.

\section{DISCUSSION}

Here, in the first analysis assessing blood biomarkers in RIC in acute ischaemic stroke patients, it has been demonstrated that RIC reduced SAP and TNF- $\alpha$ levels. Overall, increasing SAP levels pre- to post-intervention and pre-intervention to day 4 were significantly moderately correlated with worse clinical outcomes after ischaemic stroke. Further, higher S100- $\beta$ levels at pre-intervention, postintervention and day 4 were significantly moderately correlated with worse NIHSS scores at day 90 . No other biomarkers had significant and consistent correlations with outcome at day 90 .

In the primary RECAST-1 publication a significant increase in total and phosphorylated HSP 27 was reported in the RIC group, whilst other HSPs $(60,70)$ and C-reactive protein, S100- $\beta$, MMP9 , troponin $\mathrm{T}$ and endocannabinoid levels did not differ between treatment groups [4]. These data are added to by demonstrating that RIC was associated with reductions in SAP and TNF- $\alpha$ pre- to post-intervention but did not influence other inflammatory cytokines or putative neuroprotective biomarkers. The effect of RIC on reducing SAP and TNF- $\alpha$ was not maintained at day 4 . This may indicate that single four-cycle RIC might be insufficient to reduce these 


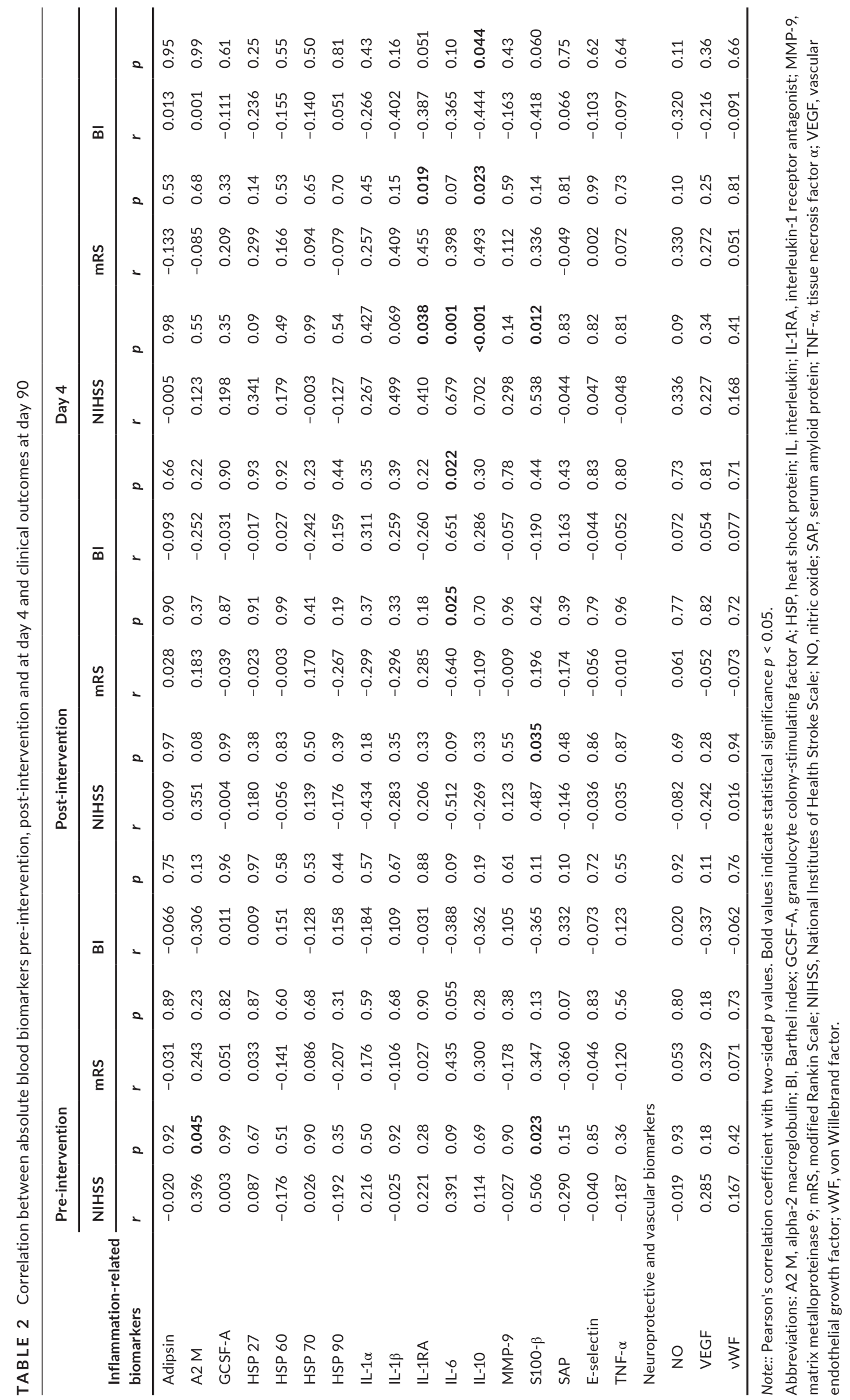




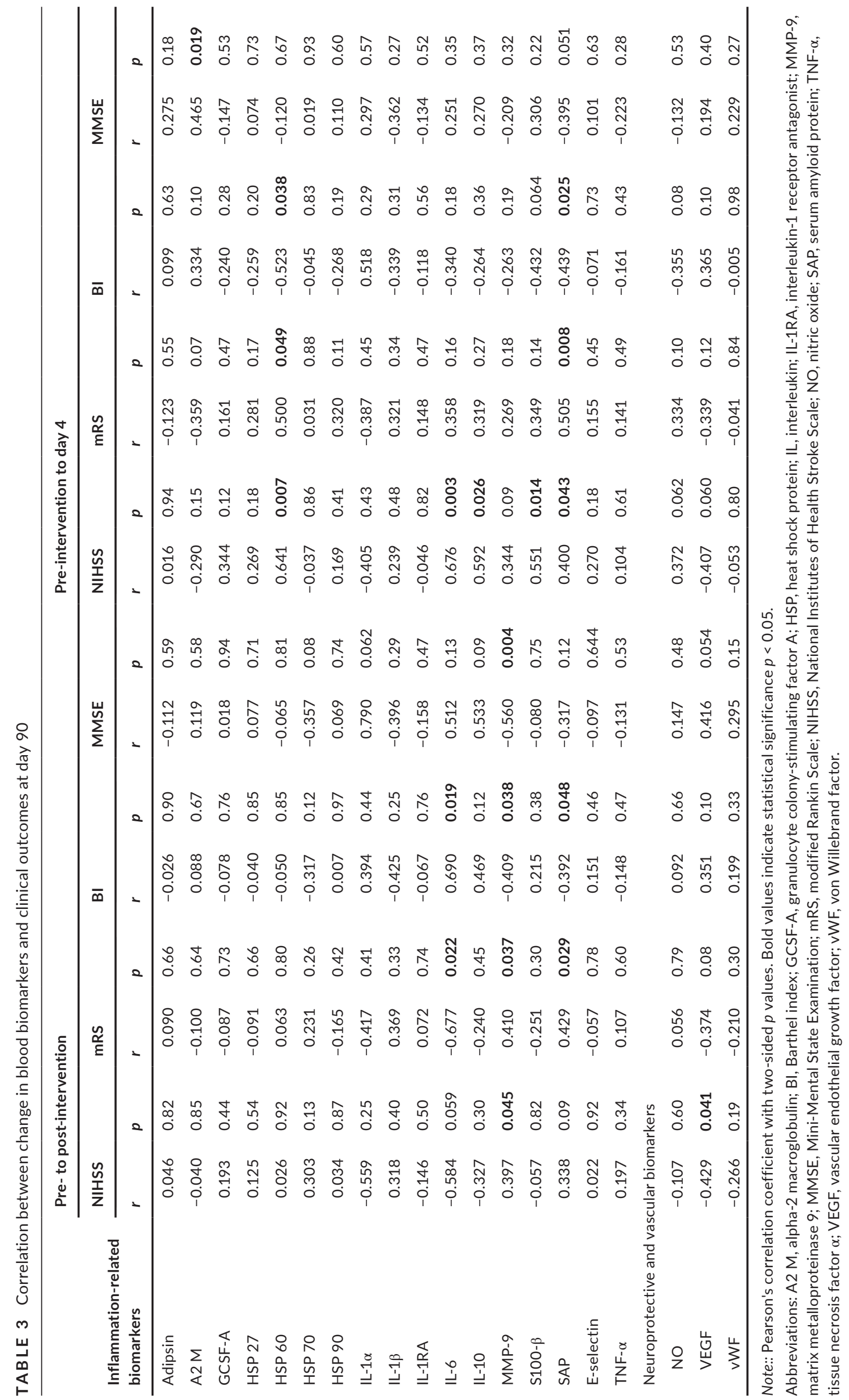


pro-inflammatory markers over a longer period. Whether repeated cycles of RIC over the first days prolong the reduction in these pro-inflammatory markers is a question for future studies.

In contrast to the present study, data from 50 healthy volunteers revealed that RIC was associated with elevated VEGF and four inflammation-related markers (transforming growth factor $\beta 1$, leukaemia inhibitory factor, MMP-9 and tissue inhibitor of metalloproteinases 1) $1 \mathrm{~h}$ post-RIC [11]. These findings in healthy volunteers cannot be extrapolated to acute stroke patients. However, a recent study assessing RIC five times a week for 8 weeks after acute ischaemic stroke (post-conditioning) found that those who received RIC had lower levels of nuclear factor $\kappa B$ (an inflammatory transcription factor) and toll-like receptor 4 (a mediator of systemic inflammatory response) and improved cerebral collateral circulation scores on computed tomography angiography [12]. This anti-inflammatory effect of RIC was also seen in older patients with symptomatic intracranial arterial stenosis who received 180 days of bilateral arm ischaemic preconditioning resulting in reduced markers of inflammation including highly sensitive C-reactive protein and IL-6 [13]. Here, it has been demonstrated that RIC administered as a one-off dose of four 5-min cycles of limb ischaemia may reduce some pro-inflammatory markers whilst promoting HSP 27 which may prevent breakdown of the bloodbrain barrier [14].

An increase in SAP from pre- to post-intervention and pre-intervention to day 4 was associated with worse clinical outcomes at day 90 , including cognition, across the trial population. SAP has an important role in inflammation and innate immunity, activating the classical complement pathway with increased SAP levels being associated with increased death at day 90 after acute ischaemic stroke [6]. SAP binds to all forms of amyloid fibrils, is present in amyloid deposits and prevents proteolysis of the amyloid fibrils of Alzheimer disease [15]; its role in vascular cognitive impairment, however, is not well understood. Nonetheless, proof-of-concept studies of vascular cognitive impairment and RIC demonstrate twice daily RIC for 1 year improved visuospatial and executive function and reduced white matter hyperintensities compared with sham in a small study of 30 patients with cerebral small vessel disease [16]. One putative mechanism is that RIC reduces pro-inflammatory biomarkers (such as SAP), thereby improving vascular health and building ischaemic tolerance, resulting in improved clinical outcomes including cognition. Indeed, repeated RIC (or chronic RIC) has beneficial effects on vascular and endothelial function in healthy volunteers [17] and on cerebral blood flow after stroke caused by intracranial stenosis [18] and may be of therapeutic use in treating chronic inflammation associated with atherosclerotic disease [19].

There was no difference in change in NO levels between those randomized to RIC vs. sham. This is in contrast to the human pre-clinical literature evaluating repeated RIC on blood flow, where it is apparent that RIC enhances peripheral endothelial function through an endothelium-dependent mechanism, which is inhibited by administration of an NO synthase inhibitor [10]. This does not necessarily mean that RIC does not influence NO levels in ischaemic stroke; it may suggest that the intervention occurred too late after stroke onset to influence NO levels, or repeated doses are needed, or the analyses are underpowered. In addition, NO has a very short half-life and so is challenging to measure. RIC has been assessed in other small studies involving ischaemic stroke patients within the thrombolysis window $[20,21]$ in the context of mechanical thrombectomy [22] and in planned larger phase III efficacy trials including RECAST-3 (ISRCTN63231313) and Remote Ischemic Conditioning in Patients With Acute Stroke (RESIST, NCT03481777).

There are a number of limitations in our study. First, it was powered for tolerability of the intervention and not to detect changes in biomarkers; hence, the biomarker analyses may be underpowered. Further, the associations seen here may represent chance, perhaps in part due to multiple testing, and require confirmation in larger cohorts. In these exploratory and hypothesis-generating analyses, two putative biomarkers were positively influenced by RIC and reassuringly there were no concerning safety signals from this dataset. Secondly, participants were recruited $16 \mathrm{~h}$ after stroke onset, which may be too late for RIC to exert any potential beneficial effect. Other studies have randomized patients in the hyperacute period and blood biomarker comparison with the present study may prove illuminating and instructive. Thirdly, RECAST-1 performed one set of four cycles of RIC and it is unclear how many cycles of RIC and over what time period are optimal; the following RECAST-2 trial addressed this [20]. Last, advanced imaging data are not available to quantify lesion size, which may have potentially influenced our findings within or between treatment groups.

In summary, increases in plasma levels of SAP are associated with worse clinical outcomes after ischaemic stroke. RIC reduced SAP and TNF- $\alpha$ levels from pre- to post-intervention. These findings should be considered preliminary given the size of the study. Larger studies assessing biomarkers, safety and efficacy of RIC in acute ischaemic stroke are warranted; the RECAST-3 (ISRCTN63231313) and RESIST (NCT03481777) trials are addressing this question.

\section{CONFLICT OF INTERESTS}

JPA was supported, in part, by NIHR HTA TARDIS and BHF RIGHT-2 trials. PMB is Stroke Association Professor of Stroke Medicine, is an NIHR Senior Investigator, reports grants from British Medical Association, NIHR, BHF, and personal fees from Moleac, Phagenesis, DiaMedica, Sanofi, Nestle outside the submitted work. TE is the Chief Investigator of NIHR EME-funded RECAST-3. The remaining authors report no disclosures.

\section{AUTHOR CONTRIBUTIONS}

JPA performed some of the laboratory analyses, statistical analyses, wrote and edited the manuscript. SO'S performed some of the laboratory analyses and edited the manuscript. $\mathrm{AH}$ performed data collection, project administration and edited the manuscript. JAM performed some of the laboratory analyses and edited the manuscript. RD, NS and PB edited the manuscript. TE conceptualized the study, gained funding, supervised the project, edited the manuscript and is overall guarantor of the study. 


\section{FUNDING INFORMATION}

RECAST-1 was funded by the British Medical Association Vera Down grant. JPA was supported, in part, by the National Institute of Health Research (NIHR) HTA TARDIS and BHF RIGHT-2 trials.

\section{DATA AVAILABILITY STATEMENT}

The data that support the findings of this paper are available from the corresponding author upon reasonable request.

\section{ORCID}

Jason P. Appleton (D) https://orcid.org/0000-0002-2604-4410

Saoirse E. O'Sullivan (D) https://orcid.org/0000-0002-1672-6610

Nikola Sprigg (D) https://orcid.org/0000-0002-5871-8168

Philip M. Bath (D) https://orcid.org/0000-0003-2734-5132

Timothy J. England (D) https://orcid.org/0000-0001-5330-8584

\section{REFERENCES}

1. Chen G, Thakkar M, Robinson C, Doré S. Limb remote ischemic conditioning: mechanisms, anesthetics, and the potential for expanding therapeutic options. Front Neurol. 2018;9:40.

2. Hess DC, Blauenfeldt RA, Andersen G, et al. Remote ischaemic conditioning-a new paradigm of self-protection in the brain. Nat Rev Neurol. 2015;11:698.

3. Weir P, Maguire R, O'Sullivan SE, England TJ. A meta-analysis of remote ischaemic conditioning in experimental stroke. J Cereb Blood F Metab. 2020;0271678X2092407 https://doi.org/10.1177/02716 $78 \times 20924077$

4. England TJ, Hedstrom A, O'Sullivan S, et al. RECAST (Remote Ischemic Conditioning After Stroke Trial): a pilot randomized placebo controlled phase II trial in acute ischemic stroke. Stroke. 2017;48(5):1412-1415.

5. Prugger C, Luc G, Haas B, et al. Adipocytokines and the risk of ischemic stroke: the PRIME study. Ann Neurol. 2012;71(4):478-486.

6. Gori AM, Giusti B, Piccardi B, et al. Inflammatory and metalloproteinases profiles predict three-month poor outcomes in ischemic stroke treated with thrombolysis. J Cereb Blood Flow Metab. 2017;37(9):3253-3261.

7. Yilmaz G, Granger DN. Cell adhesion molecules and ischemic stroke. Neurol Res. 2008;30(8):783-793.

8. Greenberg DA, Jin K. Vascular endothelial growth factors (VEGFs) and stroke. Cell Mol Life Sci. 2013;70(10):1753-1761.

9. De Meyer SF, Stoll G, Wagner DD, Kleinschnitz C. von Willebrand factor: an emerging target in stroke therapy. Stroke. 2012;43(2):599-606.

10. Kimura M, Ueda K, Goto C, et al. Repetition of ischemic preconditioning augments endothelium-dependent vasodilation in humans: role of endothelium-derived nitric oxide and endothelial progenitor cells. Arterioscler Thromb Vasc Biol. 2007;27(6):1403-1410.

11. Guo Z-N, Guo W-T, Liu J, et al. Changes in cerebral autoregulation and blood biomarkers after remote ischemic preconditioning. Neurology. 2019;93(1):e8.
12. Ji Z, Yu L, Wu W, Fang Q. Remote ischemic postconditioning promotes collateral circulation and down-regulates TLR4/NF- $\mathrm{KB}$ signaling pathway in patients with acute ischemic stroke. Int J Exp Clin Med. 2020;13(1):246-252.

13. Meng R, Ding $Y$, Asmaro $K$, et al. Ischemic conditioning is safe and effective for octo- and nonagenarians in stroke prevention and treatment. Neurotherapeutics. 2015;12(3):667-677.

14. Shi $Y$, Jiang $X$, Zhang L, et al. Endothelium-targeted overexpression of heat shock protein 27 ameliorates blood-brain barrier disruption after ischemic brain injury. Proc Natl Acad Sci. 2017;114(7): E1243

15. Tennent GA, Lovat LB, Pepys MB. Serum amyloid P component prevents proteolysis of the amyloid fibrils of Alzheimer disease and systemic amyloidosis. Proc Natl Acad Sci U S A. 1995;92(10): 4299-4303.

16. Wang $\mathrm{Y}$, Meng $\mathrm{R}$, Song $\mathrm{H}$, et al. Remote ischemic conditioning may improve outcomes of patients with cerebral small-vessel disease. Stroke. 2017;48(11):3064-3072.

17. Jones H, Hopkins N, Bailey TG, Green DJ, Cable NT, Thijssen DH. Seven-day remote ischemic preconditioning improves local and systemic endothelial function and microcirculation in healthy humans. Am J Hypertens. 2014;27:918-925.

18. Meng R, Asmaro K, Meng L, et al. Upper limb ischemic preconditioning prevents recurrent stroke in intracranial arterial stenosis. Neurology. 2012;79:1853-1861.

19. Chong J, Bulluck H, Fw Ho A, Boisvert WA, Hausenloy DJ. Chronic remote ischemic conditioning for cardiovascular protection. Cond Med. 2019;2:164-169.

20. England TJ, Hedstrom A, O'Sullivan SE, et al. Remote ischemic conditioning after stroke trial 2: a phase Ilb randomized controlled trial in hyperacute stroke. J Am Heart Assoc. 2019;8(23):e013572.

21. Pico F, Rosso C, Meseguer E, et al. A multicenter, randomized trial on neuroprotection with remote ischemic per-conditioning during acute ischemic stroke: the REmote iSchemic Conditioning in acUtE BRAin INfarction study protocol. Int J Stroke. 2016;11(8): 938-943.

22. Zhao W, Che R, Li S, et al. Remote ischemic conditioning for acute stroke patients treated with thrombectomy. Ann Clin Transl Neurol. 2018;5(7):850-856.

\section{SUPPORTING INFORMATION}

Additional supporting information may be found online in the Supporting Information section.

How to cite this article: Appleton JP, O'Sullivan SE,

Hedstrom A, et al. Blood markers in remote ischaemic conditioning for acute ischaemic stroke: data from the REmote ischaemic Conditioning After Stroke Trial. Eur J Neurol. 2020;00:1-9. https://doi.org/10.1111/ene.14650 